\title{
Screening and Identification of LncRNAs Related to Villus Growth of Liaoning Cashmere Goats and Their Effects on Growth after FGF5 Treatment
}

Mei Jin ( $\sim$ jm6688210@163.com )

Liaoning Normal University https://orcid.org/0000-0001-7986-5020

Qin Feng Zhao

Liaoning Normal University

Ping Ni

Liaoning Normal University

Jun Piao

Liaoning Normal University

Ai Jing Piao

Liaoning Normal University

Research article

Keywords: cashmere, FGF5, long non-coding RNA, keratin associated protein

Posted Date: July 29th, 2020

DOI: https://doi.org/10.21203/rs.2.13339/v4

License: (c) (1) This work is licensed under a Creative Commons Attribution 4.0 International License.

Read Full License 


\section{Abstract}

(Background)Liaoning Cashmere Goat cashmere has high economic value FGF5 is an important factor regulating its growth. The role of long non-coding RNA (LncRNA) in the mammalian villus growth cycle has still not been studied in detail.

(Results)We demonstrated that treatment of skin cells with FGF5 inhibited the expression of LncRNA in cells, down-regulated the expression of the target genes CBS and CTH, and promoted the expression of related keratin genes k26, kap11.1. Overexpressing LncRNA reversed the inhibiting effect of FGF5 on the target genes CBS and CTH.

(Conclusions)we believe that FGF5 can regulate the growth and development of Cashmere Goat hair by promoting the expression of related keratin and keratin-associated protein genes. This mechanism is achieved by inhibiting the expression of the LncRNA gene and then down-regulating the expression of the target genes CBS and CTH.

\section{Background}

The Liaoning Cashmere Goat is a unique breed in China whose wool and meat have high functional value.Liaoning Cashmere Goat wool is of high quality and fluff yield. It is a very precious textile fiber and has the reputation of being the "fiber gem"[1-2]. In mammals, hair is a skin derivative that grows and develops from hair follicles: one of the most striking features of hair follicles is that they self-renew throughout the life of an individual and continue to produce new hair[3].

Hair follicles are an important structure for regulating villus growth. There are two main types - primary and secondary hair follicles-and the primary hair follicles can be further differentiated into secondary hair follicles. The primary hair follicle produces hair fibers, and the secondary hair follicle produces velvet fibers [4]. The quality of the fluff is determined by the velvet fibers. The growth and development of hair follicles is a cyclical physiological process that undergoes three regular periods in a year: anagen, catagen, and telogen [5-8]. Many factors - such as endocrine hormones and drug treatment of skin cellscan affect this cycle, which in turn affects the growth of cashmere.

Fibroblast growth factor 5 (FGF5) can regulate the hair follicle from the anagen to the telogen period and change the growth cycle of hair follicle cells. FGF5 is a cell growth factor obtained and purified from the mammalian pituitary gland. It has been found that FGF5 can advance the hair follicle growth cycle into the telogen period and inhibit hair growth [9]. Wichert B et al. found that mutations in the FGF5 gene can cause genetic hair length variation in mice and dogs [10]. Hebert's study found that, if the mouse FGF5 gene is silenced, the mouse hair follicle anagen will be prolonged, and the mouse hair will be longer, which prolongs the telogen of the hair follicle [11]. The FGF5 gene is also a determinant of the long hair phenotype in domestic cats. The long subtype of the FGF5 gene in alpacas prematurely produces a point mutation in the stop codon (PTC), leading to an increase in alpaca hair length [12]. Steven Krege found that Sox2 can regenerate hair follicle cells in the skin through FGF5[13-14]. The above results indicate 
that FGF5 factor plays a very important role in the cyclical changes of hair follicles and the growth and development of villi. Therefore, research on FGF5 in hair follicle development and villus growth is very important.

With the deepening of scientific research, many long non-coding RNAs (LncRNAs) have been shown to have a certain regulatory effect on the metabolism and growth of living organisms[15]. LncRNAs regulation mechanism and participating biological processes mainly include chromosome modification, regulation of transcripts (up- or down-regulation of gene expression), localization of subcellular proteins, cell proliferation and differentiation, immune response mechanisms, tumor cancer, etc.[16-17]. LncRNAs also regulates genes involved in hair follicle development and the postnatal hair cycle[18]. Cai et al. analyzed the regulation of LncRNA AK015322 (LncRNA5322) in hair follicle stem cells (HFT) and the potential mechanism of IncRNA5322-mediated HFT differentiation. The results showed that LncRNA5322 can target miR-21-mediated PI3K-AKT Signaling pathways in HFT promote the proliferation and differentiation of HFT[19]. In 2017, the non-coding RNA database LncRNASNP2

(http://bioinfo.life.hust.edu.cn/LncRNASNP2) began providing comprehensive information on LncRNA mutations, as well as the structure and function of LncRNAs. The database contains 141,353 human and 117,405 mouse LncRNAs. Scholars identified LncRNAs associated with growth in cows [20-21].

Even though research on hair follicle development and LncRNAs is increasing, genetic information on Cashmere Goats remains relatively small, and there are few known genes related to the growth and development of villi. We believe that some LncRNAs may play an unknown role in the villi development of Cashmere Goats. This study investigated the effect of LncRNA on the growth and development of villi in skin cells treated with FGF5 and suggests a new way to approach the study of cashmere growth in Cashmere Goats.

Villi formation in Cashmere Goats has been found to require keratin and the keratin-associated protein (KAP) family. Reduction of the keratin K25 gene can cause hair loss [22]; the K17 gene can change the growth cycle of villi [23]; the KAP family is more abundant in mammalian hair follicles, and some keratin genes play a role in the differentiation of hair tissue [24-25]. It has been found that the KAP16.6 and KAP13.1 genes are involved in regulating the size of the villous fiber diameter in sheep [26-27]. The KAP18 gene plays an important role in maintaining flexibility and structural integrity in epithelial cells [2829]. The keratin-associated protein 13.3 is composed of 156 amino acids, and the Cashmere Goat KAP13.3 gene may affect the structure of this protein and characteristics of cashmere fibers [30-31]. KAP is the main component of wool, and keratin and keratin-associated proteins are composed of amino acids.

The body's complex regulation activities, like cell metabolism, are closely related to the amino acid metabolic pathway; Biolo $G$ et al. found that, when the body's activity is reduced, the stimulation of amino acid synthesis on the body's protein is greatly reduced [32]. Some amino acids have been shown by scientists to be related to the growth and development of hair follicles. Asparagine plays an important role in the regulation of hair follicle growth and development [33]. By supplementing the cysteine amino 
acid, cells can resist the decrease in keratin synthesis caused by the loss of iron, which affects the growth and development of hair follicles [34]. Sulfur-containing amino acids (cysteines) are critical for the structural maintenance of keratin and keratin-associated proteins, which are also rich in serine [35]. In summary, we can find that the KAP family and amino acid metabolic pathway play an important role in the regulation of hair follicle development and villus quality. Therefore, it is very important to study the KAP family to regulate the growth of villi.

The villi in the Liaoning Cashmere Goat studied in this experiment are composed of about $90 \%$ keratin and keratin-associated proteins. We found that LncRNA is involved in the amino acid metabolic pathway and plays a regulatory role in synthesizing serine and cysteine. For the KAP family, sulfur-containing amino acids (methionine and cysteine, which can be converted into each other) play an important role in their structural maintenance. Therefore, we believe that FGF5 can affect the production of members of the keratin family in Cashmere Goat skin cells by regulating the expression of LncRNA, further affecting the growth and development of Cashmere Goat villi and the quality of villi.

\section{Results}

\section{Screening differentially-expressed LncRNA}

The total RNA in a sample was subjected to mass detection using an Agilent Technologies 2100 Bioanalyzer and a NanoDrop 1000 spectrophotometer, and coverage analysis of different known gene types of the species samples was performed using HTSeq software. The results of differential expression analysis of LncRNA are shown in Table 1. (screening threshold is Qvalue < 0.05):

Table 1. Screening results of differentially-expressed LncRNA

\begin{tabular}{cccc}
\hline Sample & $\begin{array}{c}\text { Total number of LncRNA of } \\
\text { differential expression }\end{array}$ & $\begin{array}{c}\text { Total number of LncRNA of } \\
\text { up-regulation expression }\end{array}$ & $\begin{array}{c}\text { Total number of LncRNA of } \\
\text { down-regulation expression }\end{array}$ \\
\hline $\begin{array}{c}\text { F4_24h } \\
\text { Vs C }\end{array}$ & 164 & 70 & 94 \\
$\begin{array}{c}\text { F4_72h } \\
\text { Vs C }\end{array}$ & 189 & 78 & 111 \\
F6_24h & 123 & 27 & 96 \\
Vs C & & & \\
\hline
\end{tabular}


The above results showed that F4_24h and C were screened to obtain 164 differentiallyexpressed LncRNA, of which 70 were up-regulated and 94 were down-regulated; F4_72h was compared to C, and there were 189 differentially-expressed LncRNA, of which 78 were up-regulated and 111 were down-regulated; F6_24h was compared to C, 123 differentiallyexpressed LncRNA were obtained, of which 27 were up-regulated and 96 were downregulated.

\section{Functional enrichment analysis of differentially-expressed LncRNA target genes}

After differentially-expressed LncRNA were predicted by cis and trans target genes, GO and KEGG functional enrichment analyses were performed on their target genes, and the screening conditions were corrected $(\mathrm{P}<0.05)$. GO analysis showed that the cis and trans target genes of LncRNA differentially-expressed in F4_24h and C groups had no significant enrichment of the GO term; the cis target genes of LncRNA differentially-expressed in F4_72h and C groups had no significant enrichment of the GO term; The trans target gene was mainly enriched in the cellular metabolic process biological_process and cellular macromolecule metabolic process biological_process of BP; cell cellular_component, cell part cellular_component and intracellular cellular_component of CC; and binding molecular_function of MF. The cis and trans target genes of LncRNA differentiallyexpressed in the F6_24h and C groups also had no significantly enriched GO term. The above results indicated that the GO enrichment results were most significant in the F4_72h treatment group (Fig. 1).

The KEGG pathway enrichment analysis was performed on the target genes of differentially-expressed LncRNA in the three groups (screening conditions: Qvalue < 0.05). The results are shown in Table 2.

Table 2. KEGG enrichment analysis results 


\begin{tabular}{|c|c|c|c|}
\hline \multicolumn{2}{|c|}{ Sample name } & Significantly enriched pathway term & Qvalue \\
\hline \multirow[t]{2}{*}{ F4_24h vs C } & Cis target gene & - & - \\
\hline & Trans target gene & - & - \\
\hline \multirow[t]{9}{*}{ F4_72h vs C } & Cis target gene & - & - \\
\hline & Trans target gene & Ribosome & 0.001 \\
\hline & & RNA transport & 0.018 \\
\hline & & Fanconi anemia pathway & 0.018 \\
\hline & & Huntington's disease & 0.018 \\
\hline & & Metabolic pathways & 0.025 \\
\hline & & Aminoacyl-tRNA biosynthesis & 0.029 \\
\hline & & Citrate cycle (TCA cycle) & 0.033 \\
\hline & & Alzheimer's disease & 0.033 \\
\hline F6_24h vs C & Cis target gene & $\begin{array}{l}\text { Ubiquitin mediated proteolysis } \\
\text { Taste transduction }\end{array}$ & $\begin{array}{l}0.037 \\
0.022\end{array}$ \\
\hline & Trans target gene & - & - \\
\hline
\end{tabular}

The above results indicate that the pathway enrichment results were most significant in the F4_72h treatment group, which was most closely related to hair follicle development and villus growth. Based on the above results and by consulting the literature, it was determined that metabolic pathways are involved in the regulation of hair follicle development and villus growth.

\section{Expression verification of target LncRNA}

Combined with the screening of LncRNA, prediction of target genes, and bioinformatics analysis of LncRNA, it was finally determined that FGF5 had the most beneficial effects on hair follicle development and villus growth when it treated Liaoning Cashmere Goat skin cells with $10^{-4} \mathrm{~g} / \mathrm{L}$ for $72 \mathrm{~h}$. Therefore, in the experiment, we screened four LncRNAs related to the action of FGF5 from the $10^{-4} \mathrm{~g} / \mathrm{L} 72 \mathrm{~h}$ treatment group: XLOC_011424, XLOC_009522, XLOC_009063, XLOC_011157. To further determine the accuracy of RNA- 
Seq results, we utilized the qPCR technique to detect the expression of the above four LncRNAs, as shown in Fig. 2 to Fig. 5.

The results showed that, after treating Liaoning Cashmere Goat skin cells with FGF5, the expression of LncRNA XLOC_011424 was down-regulated $(\mathrm{P}<0.05)$ to 0.70 times that of the control group; the expression of LncRNA XLOC_009063 was up-regulated $(\mathrm{P}<0.05)$ to 1.12 times that of the control group; and the expression of LncRNA XLOC_011157 was down-regulated $(\mathrm{P}<0.05$ ) to 0.74 times that of the control group. The results of downregulation of the expression of the four LncRNAs based on RNA-Seq sequencing indicated that LncRNA XLOC_011424 and LncRNA XLOC_011157 were consistent with previous results. We first selected LncRNA XLOC_011424 as the target LncRNA to study its mechanism of action on fluff growth after treating Liaoning Cashmere Goat skin cells with FGF5.

\section{qPCR detection of k26, kap11.1 gene expression in cells after FGF5 drug treatment}

After treating Cashmere Goat skin cells with FGF5, the expression levels of k26, kap11.1 genes were detected by qPCR (Fig. 6). The results showed that, compared to untreated cells, the cells with keratin26 genes that were treated with FGF5 were upregulated at the mRNA level, and the expression level was increased. There was no significant change in the expression of the kap11.1 gene. This indicated that FGF5 played a positive role in the regulation of keratin-related genes k26 but has no clear effect on the keratin-related gene kap11.1 was observed.

\section{FISH immunofluorescence localization}

The location of the target LncRNA in the skin cells of Cashmere Goats was studied by the FISH immunofluorescence localization technique (blue staining reagent marks the nucleus, red staining reagent marks the target LncRNA, and overlapped blue and red stain 
values refer to a mixture of the two). According to the results of this experiment, LncRNA is mainly localized in the nucleus and cytoplasm in the hair cells of Cashmere Goats. After cells were treated with FGF5, it was found that FGF5 induced LncRNA out of the nucleus, where it mainly became localized in the cytoplasm.(Fig. 7)

\section{Detection of the LncRNA target gene and related keratin expression in Liaoning CashmereGoat fibroblasts after LncRNA overexpression}

In order to study the effect of LncRNA overexpression on velvet growth-related genes, an LncRNA overexpression vector was constructed by lentiviral-mediated technology and successfully transfected into Cashmere Goat skin fibroblasts. Treating skin cells with FGF5 yielded the following: FGF5 treatment inhibited the expression of LncRNA in cells, downregulated the expression of the target genes CBS and $\mathrm{CTH}$, and promoted the expression of related keratin genes $\mathrm{k} 26$, kap11.1. In order to study the effect of LncRNA on the expression of keratin and keratin-associated proteins-and to construct the relationship between FGF5, LncRNA and KAP family-we overexpressed LncRNA with FGF5 treatment which reversed the inhibiting effect of FGF5 on the target genes CBS and CTH; this further inhibited the expression of k26, kap11.1 in the cells (Fig. 8-9).

\section{Detection of the LncRNA target gene and related keratin expression in Liaoning Cashmere Goat fibroblasts after LncRNA interference}

An LncRNA interference vector was constructed by lentiviral-mediated technology and successfully transfected into Cashmere Goat skin fibroblasts. This down-regulated the expression of LncRNA in skin fibroblasts, and the expression of interfering lentiviral vector was detected by qPCR. The results showed that the interference target spots of 31, 131, 231 were significant, and the 131 target spot interference effect reaches $60 \%$, so

interference target spot 131 was used for subsequent experiments (Fig. 10). After treatment of skin cells with FGF5, qPCR showed that, when the expression level of LncRNA gene was 
decreased, the expression levels of the target genes CBS and CTH were inhibited while the expression levels of LncRNA and target genes decreased. The expression levels of the k26, kap11.1 genes all increased (Fig. 11). This indicates that the LncRNA gene plays a negative regulatory role in the function of keratin and keratin-associated protein genes.

\section{Discussion}

Liaoning Cashmere Goats are known for their high-quality cashmere and stable genetics. Hair follicle development and villus growth are affected by many factors, one of which is FGF5, a cytokine found to be directly related to hair length [36]. Since the cause of the growth of the Angora mouse hair was discovered, people have been working on the mechanism by which FGF5 influences hair follicle development and villus growth. However, people have been focused on the study of hair follicle development and villus growth related genes, ignoring most effects of non-coding RNA. In recent years, people have begun to pay attention to the regulation of non-coding RNA in various life activities, including hair follicle development and villus growth.

In this study, the FGF5-treated Liaoning Cashmere Goat skin cells were sequenced by RNA-seq sequencing technology, and LncRNA related to hair follicle development and villus growth were screened. We first selected LncRNA XLOC_011424 (LncRNA) for research and established the association between LncRNA and the target genes CBS, CTH, keratin K26 and the keratin-associated proteins KAP11.1. We worked toward explaining the effect of LnCRNA XLOC_011424 on regulating hair follicle cycle development and villus growth under FGF5 treatment.

In addition, results of the experiment showed that amino acid metabolism in the cell metabolic pathway is related to hair follicle development and villus growth. It has been reported that amino acid types and contents can affect hair follicle development and villus growth; Miniaci MC's research found that cysteine is related to the expression of keratin, meaning it affects the development and maturation of hair follicles [37]. Studies have shown that the development of hair follicles and the growth of villi are affected by the type and content of amino acids, that is, the amino acid metabolism pathway can affect the development of hair follicles and the growth of villi.

In this experiment, we found that the target genes corresponding to LncRNA XLOC_011424 are distributed in the amino acid metabolic pathway, which is part of the cellular metabolic pathway, mainly involved in the conversion between serine and cysteine, serine and threonine, indicating indirectly that these amino acids are involved in hair follicle development and regulation of villus growth. We found that cysteine and serine are abundant in keratin and keratin-associated proteins, which are the main components of hair fibers. In the regulation of FGF5 on hair follicle development and villus growth, FGF5 synthesizes cysteine and serine by affecting LncRNA regulation of amino acid metabolism pathway, thereby affecting the expression of keratin and keratin-associated proteins, ultimately affecting hair follicle development and villi growth. In this experiment, interference lentiviral technology and overexpression lentivirus 
technology were used to clarify the regulation of LncRNA in villus growth and development. The results showed that LncRNA under the action of FGF5 further promotes Cashmere Goat hair follicle growth and development by regulating the expression of target genes and related keratin family genes.

In summary, this study found for the first time that LncRNA is related to the FGF5 regulation of hair follicle development and villus growth; it also demonstrates that the amino acid metabolism pathway is related to hair follicle development and villus growth to some extent. The mechanism by which FGF5 regulates hair follicle development and villus growth by overexpression and interference lentivirus technology further lays a theoretical foundation for molecular-assisted breeding of Liaoning Cashmere Goats.

\section{Conclusion}

We demonstrated that treatment of skin cells with FGF5 inhibited the expression of LncRNA in cells, down-regulated the expression of the target genes CBS and CTH, and promoted the expression of related keratin genes k26, kap11.1. Then, overexpressing LncRNA in skin cells reversed the inhibiting effect of FGF5 on the target genes CBS and CTH which further inhibited the expression of k26, kap11.1. Finally, we demonstrated the inhibition of CBS and CTH and elevation k26 and kap11.1 genes when the expression level of LncRNA gene is downregulated using RNA interference.Therefore, we believe that FGF5 can regulate the growth and development of Cashmere Goat hair by promoting the expression of related keratin and keratin-associated protein genes. This mechanism is achieved by inhibiting the expression of the LncRNA gene and then down-regulating the expression of the target genes CBS and CTH.

\section{Methods}

\section{Collection of experimental samples and FGF5 drug treatment}

The research protocol of this experiment was approved by the Animal Protection and Use Committee of the College of Life Sciences of Liaoning Normal University and the Chinese Agriculture Committee. The animal and experimental operations involved were guided by the animal protection and treatment system.

In mid-September, six adult male Liaoning Cashmere Goats (males have a high cashmere yield) were randomly selected from the Liaoning Cashmere Goat Production Base (Dalian Wafangdian City, Liaoning Province). They were locally anesthetized with 5\% procaine (Sangon, Shanghai, China) before skin biopsy. then the subcutaneous adipose tissue was removed, cut into pieces under sterile conditions and cultured with cell tissue adherent method. After the small pieces of tissue adhere to the wall, add 10ml of DMEM 
medium containing $20 \%$ FBS to the bottom of the bottle. After 2-3 days of culture, when the cells grow to $70 \%-80 \%$ confluence, add trypsin to digest. When the cells become round Then, add DMEM medium containing10\% FBS to terminate the digestion. The bottom of the bottle was repeatedly blown with a pipette to make the cells fall off the bottom. The cell mixture was transferred to a centrifuge tube and centrifuged. The supernatant was discarded. $2 \mathrm{ml}$ of DMEM containing 20\% FBS party was added to the centrifuge tube, and then the cells were repeatedly blown $\llbracket$ divide cells evenly into two flasks, add $7 \mathrm{ml}$ of DMEM culture solution containing $20 \% \mathrm{FBS}$, and place them in a $37^{\circ} \mathrm{C} 5 \% \mathrm{CO}_{2}$ incubator for cultivation.

\section{Screening of target LncRNA and functional enrichment analysis of target genes}

Total RNA was purified from Goat fibroblasts with TRIzol (TaKaRa, Dalian, China). The quality of the total RNA was detected with a NanoPhotometer ${ }^{\circledR}$ spectrophotometer (Implen, Westlake Village, CA, USA), and RNA samples were treated with DNase I (Bao Bioengineering (Dalian) Co. Ltd.). The total RNA quality was detected and identified by agarose gel electrophoresis, NanoDrop1000 micro UV-Vis spectrophotometer and Agilent Technologies 2100 analyzer and then a library was built, four sets of RNA-Seq library samples were sequenced and evaluated for sequencing data using the Illumina HiseqTM 2500 (Beijing Novo Zhiyuan Bioinformatics Co., Ltd.) with the PE125 sequencing strategy. The TopHat2 algorithm was used for sequence alignment analysis, and LncRNA was screened and its coding potential analyzed using CPC, CNCI, pfam protein domain and PhyloCSF analyses; the final LncRNA dataset was obtained from the intersection of these methods,Improve the reliability of the screening results. The Cncdiff (http://cufflinks.cbcb.umd.edu/manual.html\#cuffdiff) software was used to quantitatively analyze the LncRNA obtained in the experiment, screen out eligible LncRNA (P-adjust < $0.05 \square \log 2$ (Fold change) > 1), and perform cis/trans target gene prediction. We performed GO and KEGG enrichment analyses on target genes to better understand the function of differentially-expressed LncRNA target genes. The expression of the target LncRNA was then verified, the expression data of the target gene were analyzed by the 2- $\Delta \Delta$ Ct method, 
and a significant difference analysis was performed using the IBM SPSS Statistics 19 software.

\section{qPCR detection of k26, kap11.1 gene expression levels in cells after FGF5 treatment}

qPCR was used to detect the expression levels of k26, kap11.1 genes in cells after FGF5 treatment. TRIzol reagent was used to extract total RNA from samples. Total RNA samples were collected from cells treated with $10^{-4} \mathrm{~g} / \mathrm{L}$ FGF5 for $72 \mathrm{~h}$. RNA samples were treated with DNase I prior to qPCR. Total RNA in the sample was quality tested again. Reverse transcription was carried out according to the instructions of the qPCR kit (Ruisai Biotechnology Co., Ltd., Shanghai), The relative quantification of each gene expression was performed using the $\triangle \triangle \mathrm{Ct}$ method. The cDNA was stored at $-20^{\circ} \mathrm{C}$. Primers are listed in Table 3.

Table 3. qPCR detection primer information

\begin{tabular}{llc}
\hline \multicolumn{1}{c}{ Primer name } & \multicolumn{1}{c}{ Primer sequence(5'-->3') } & Products \\
\hline goat ACTB-F1 & GATGGCTACTGCTGCGTCG & 208bp \\
goat ACTB-R1 & GGCATACAGGTCCTTTCGG & \\
goat Kap11.1-F1 & CGTACCAGCAGTCCTGCGTG & $196 \mathrm{bp}$ \\
goat Kap11.1-R1 & GCCAAAGGCGGGCTTATTC & \\
goat K26-F1 & ACAACATGAGGGCTGAGTACGAG & $184 \mathrm{bp}$ \\
goat K26-R1 & TGAAGTTCTATTTCCAAGGTTTGC & \\
\hline
\end{tabular}

The total volume of the reaction system was $20 \mu \mathrm{L}$, including $10 \mu \mathrm{L}$ of $2 *$ SYBR Green Mix, $1 \mu \mathrm{L}$ of primer Mix, $5 \mu \mathrm{L}$ of template and $4 \mu \mathrm{L}$ of ultrapure water. The PCR conditions applied were pre-formed at $95^{\circ} \mathrm{C}$ for $10 \mathrm{~s}$, then subjected to 40 cycles of $95{ }^{\circ} \mathrm{C}$ denaturation for $5 \mathrm{~s}, 60{ }^{\circ} \mathrm{C}$ annealing for $30 \mathrm{~s}$, and $72{ }^{\circ} \mathrm{C}$ extension for $60 \mathrm{~s}$. 


\section{FISH fluorescence localization detection}

Experimental materials and instruments are shown in Table 4.

Table 4. FISH fluorescence localization experimental materials and instruments

\begin{tabular}{cc}
\hline Name & Manufacturer \\
\hline Liaoning cashmere goat skin fibroblast & Primary culture of cells in lab,Dalian \\
FBS & Hyclone, USA \\
EDTA Na2 & Solarbio Co., Ltd., Beijing \\
SDS & Solarbio Co., Ltd., Beijing \\
Maleic acid & Solarbio Co., Ltd., Beijing \\
Tris & Solarbio Co., Ltd., Beijing \\
LncRNA FISH Probe & Ruibo Biotechnology Co., Ltd., Guangzhou \\
Leica[Germany
\end{tabular}

Liaoning Cashmere Goat skin cell culture and FGF5 treatment was performed as previously described in this study, prepare buffer solution, different concentrations of absolute ethanol solution and DAPI solution, etc.- - was used to process the samples and carry out sample hybridization, and finally the samples were DAPI stained, sealed, observed under a confocal microscope, and photographed.

\section{Detection of expression levels of cashmere growth-related genes after LncRNA overexpression}

The target gene PCR product and target vector were digested with ASCI and Pme1 (New England Biolabs, USA), respectively. T4 DNA ligase (New England Biolabs, USA) ligated the digested PCR product and destination vector and $10 \mu \mathrm{L}$ of the ligated product was transformed into DH5 $\alpha$ competent cells (Full Golden Biotechnology Co., Ltd., Dalian). The cells were coated onto an LB plate containing ampicillin (Amp) resistance and cultured overnight at $37^{\circ} \mathrm{C}$; then positive clones were identified. Opti-MEM, expression plasmid, packaging plasmid Packaging Mix (Invitrogen, USA), POLO deliverer TM 3000 
Transfection Reagent(all from Invitrogen)were added to the culture medium of 293T cells (Jima Biotechnology Co., Ltd., Shanghai), shaken well, and cultured at $37{ }^{\circ} \mathrm{C}$ in a $5 \% \mathrm{CO}_{2}$ cell culture incubator (Likang Bios, Shanghai). The cells were collected after transfection

for a certain period of time. Finally, LncRNA overexpressing lentivirus was transfected into Cashmere Goat fibroblasts, and the expressions of LncRNA and cashmere growth-related genes were detected by qPCR.

\section{Detection of expression levels of cashmere growth-related genes after LncRNA interference}

Three interfering target spots were designed based on the target LncRNA sequence. The interference vector with the target gene was constructed as a template, Lenti-Asc1 F/Lenti -Pme1 -R were used as primers, and the EGFP-ABCA1-miR fragment was amplified by PCR, forming the enzyme cleavage sites AscI and PmeI at both ends of the target fragment. T4 DNA ligase (New England Biolabs, USA) ligated the digested PCR product and the destination vector, and transformed $10 \mu \mathrm{L}$ of the ligated product into DH5 $\alpha$ competent cells (Full Golden Biotechnology Co., Ltd., Dalian), which were coated onto an LB plate containing ampicillin (Amp) resistance, cultured overnight at $37^{\circ} \mathrm{C}$, and positive clones were identified. Opti-MEM, expression plasmid, packaging plasmid Packaging Mix (Invitrogen, USA), POLO deliverer TM 3000 Transfection Reagent were added to the culture medium of 293T cells (Jima Biotechnology Co., Ltd., Shanghai), shaken well, cultured at $37{ }^{\circ} \mathrm{C}$ in a $5 \% \mathrm{CO}_{2}$ cell culture incubator (Likang Bios, Shanghai). The cells were collected after 48h Finally, LncRNA-interfering lentivirus was transfected into Cashmere Goat fibroblasts, and the expressions of LncRNA and cashmere growth-related genes were detected by qPCR.

\section{Abbreviations}

FGF5:Fibroblast growth factor 5囚

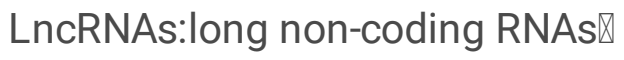

KAPs: keratin-associated proteins $\rrbracket$ 


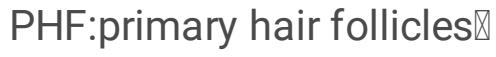

SHF:secondary hair follicles $\rrbracket$

HFT:hair follicle stem cells $\rrbracket$

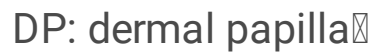

cDNA: complementary deoxyribonucleic acid》

DAPI: 4'6-diamidino-2-phenylindole区

qPCR: Real-time Quantitative PCR Detecting System $\rrbracket$

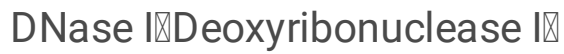

Amp囚ampicillin.

\section{Declarations}

Ethics approval and consent to participate:The study has been approved by the Ethics Committee of Liaoning Normal University, and Liaoning Cashmere Goat has obtained permission before the study. The Liaoning Provincial Key Laboratory of Biotechnology and Drug Research is responsible for ensuring the protection and care of vertebrates used in the study. We must ensure that animal research is conducted in accordance with long-term ethical principles and federal, state, and university regulations.

Consent for publication : Not Applicable

Availability of data and material: The datasets generated and/or analysed during the current study are not publicly available due Laboratory data confidentiality but are available from the corresponding author on reasonable request.

Competing interests : The authors declare that they have no competing interests.

Funding :This work was supported by National Natural Science Foundation of China [grant number 31772557]; The Foundation of Liaoning Educational Committee [grant number L201683652]; Dalian Science and Technology Innovation Fund Project [grant number 2019J12SN65]. Funding fund has made a significant contribution to our experiments. The laboratory apparatus $\square$ experimental materials and drugs 
involved in the experimental process, And participation in various academic exchange meetings are supported by the fund.

\section{Authors' contributions:}

$\mathrm{JM}$ and ZF contributed to the work equally and should be regarded as co-first authors. They are Project managers, responsible for the application, implementation, supervision, summary and acceptance of the project and the procurement of experimental consumables and drugs, cell culture, sample preparation.

NP: Responsible for statistical analysis of data.

PJ1: Responsible for collecting the latest research status of similar research topics at home and abroad.

PJ2: Responsible for the acceptance of the results of the phased experiments and the analysis and organization of data.

All authors read and approved the final manuscript.

Acknowledgements :Not applicable

\section{References}

[1] Zhu, Y.B.,Wang Z Y et al., A IncRNA-H19 transcript from secondary hair follicle of Liaoning Cashmere goat: Identification, regulatory network and expression regulated potentially by its promoter methylation[J]. Gene, 2018. 641: p. 78-85.

[2] Hu, P.F., Guan W J,Li S C,et al., Study on characteristics of in vitro culture and intracellular transduction of exogenous proteins in fibroblast cell line of Liaoning Cashmere goat[J]. Mol Biol Rep, 2013. 40(1): p. $327-36$

[3] Welle Monika M.,Wiener Dominique J.The Hair Follicle[J].Toxicologic Pathology, 2016, 44 (4):564-574.

[4] Y Dong, M Xie, Y Jiang et al. Sequencing and automated whole-genome optical mapping of the genome of a domestic goat (Capra hircus)[J]. Nat Biotechnol, 2013, 31 (2):135-41.

[5] Rogers G E. Biology of the wool follicle: an excursion into a unique tissue interaction systemwaiting to be re-discovered[J]. Experimental Dermatology, 2006, 15 (12):931-949.

[6] QL Zhang, JP Li, Y Chen et al. Growth and viability of Liaoning Cashmere goat hair follicles during the annual hair follicle cycle[J]. Genet Mol Res, 2014,13 (2):4433-43. 
[7] M Jin, CL Guo, JH Hu et al. Correlation Analysis of Economic Traits in Liaoning New Breed of Cashmere Goats Using Microsatellite DNA Markers[J]. Acta Genetica Sinica, 2006, 33 (3):230-235.

[8] GPM Moore, DL Cros, K Lsaacs et al.Hair growth induction: roles of growth factors[J]. Annals of the New York Academy of Sciences, 1991, 642 (1):308.

[9] Rogers M A, Langbein L, Winter $H$, et al. Characterization of a first domain of human high glycinetyrosine and high sulfur keratin-associated protein (KAP) genes on chromosome 21q22.1[J]. Journal of Biology Chemistry, 2002, 277 (50):48993-49002.

[10] Drögemüller, C, Rüfenacht, S, Wichert B , et al. Mutations within the FGF5, gene are associated with hair length in cats[J]. Animal Genetics, 2010, 38(3):218-221.

[11] Hébert JM, Rosenquist T, Götz J et al. FGF5 as a regulator of the hair growth cycle: evidence from targeted and spontaneous mutations[J]. Cell囚1994冈78(6):1017-1025.

[12] Pallotti S , Pediconi D , Subramanian D , et al. Evidence of post-transcriptional readthrough regulation in, FGF5, gene of alpaca[J]. Gene, 2018:S0378111918300064.

[13] Kregel S\Kiriluk KJ『Rosen AM. Sox2 is an androgen receptor-repressed gene theat promotes

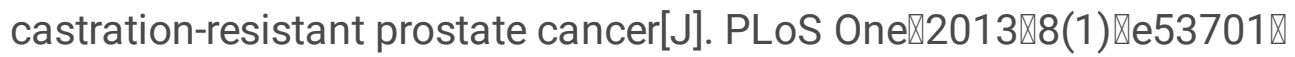

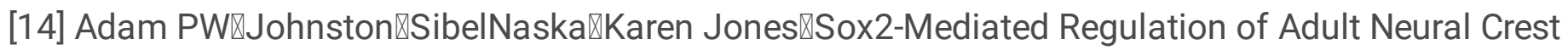
Precursors and Skin Repair[J]. Stem Cell Reports $₫ 2013 \rrbracket 1(1) \llbracket 38-45 \rrbracket$

[15] Klattenhoff CA,Scheuermann JC, Surface LE et al. Braveheart, A long noncoding RNA required for cardiovascular lineage commitment[J]. Cell. 2013, 152(3): 570-583.

[16] PC Au, QH Zhu, ES Dennis et al.Long non-coding RNA-mediated mechanisms independent of the RNAi pathway in animals and plants[J]. RNA Biol.2011,8(3):404-14.

[17] Ulitsky I , Bartel D . lincRNAs: Genomics, Evolution, and Mechanisms[J]. Cell, 2013, 154(1):26-46.

[18] Lin C M, Liu Y, Huang K, et al. Long noncoding RNA expression in dermal papilla cells contributes to hairy gene regulation. [J]. Biochemical \& Biophysical Research Communications, 2014, 453(3):508-14.

[19] Cai B, Zheng Y, Ma S, et al. Long non-coding RNA regulates hair follicle stem cell proliferation and differentiation through PI3K/AKT signal pathway[J]. Molecular Medicine Reports, 2018, 17(4):5477-5483.

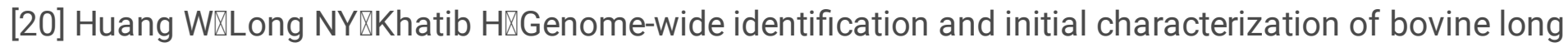
non-coding RNAs from EST data[J]. Animal Genetics $₫ 2012 \rrbracket 43(6) \llbracket 674-682 \rrbracket$

[21] Weikard R囚Hadlich F『Kuehn C『ldentification of novel transcripts and noncoding RNAs in bovine skin by deep next generation sequencing[J]. Bmc Genomics $₫ 2013 \otimes 14(1) \otimes 789 \rrbracket$ 


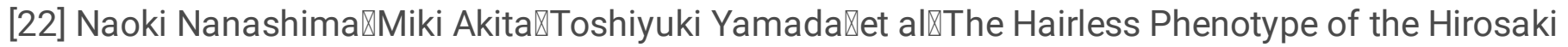
Hairless Rat Is Due to the Deletion of an 80-kb Genomic DNA Containing Five Basic Keratin Genes[J]. Journal of Biological Chemistry,2008,283(24)ه16868-16875》

[23] Tong X®Coulombe PA囚Keratin 17 modulates hair follicle cycling in a TNFa- dependent fashion[J].

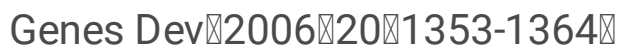

[24] Jin M $₫$ Wang L『Li S『et al『Characterization and expression analysis of KAP7.10KAP8.2 gene in Liaoning new-breeding Cashmere goat hair follicle[J]. Mol Bio Rep $₫ 2011 \rrbracket 38 \llbracket 3023-3028 \rrbracket$

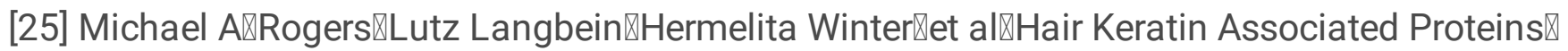
Characterization of a Second High Sulfur KAP Gene Domain on Human Chromosome 21[J]. The Journal of Investigative Dermatology $₫ 2004 \rrbracket 122 \rrbracket 147-158 \rrbracket$

[26] Meng Y, Wu Z, Yin X, et al. Keratin 18 attenuates estrogen receptor alpha-mediated signaling by sequestering LRP16 in cytoplasm[J]. BMC Cell Biology, 2009, 10 (1):1117-1132.

[27] Liffers S T, Maghnouj A, Munding J B, et al. Keratin 23, a novel DPC4/Smad4 target gene which binds 14-3-3[J]. BMC Cancer, 2011, 11 (1):137.

[28] Duncan A, Forcina J, Birt A, et al. Estrous cycle-dependent changes of Fas expression in the bovine corpus luteum: influence of keratin 8/18 intermediate filaments and cytokines[J]. Reproductive Biology and Endocrinology, 2012,10 (1): 487-487.

[29] Eriksson J E, Dechat T, Grin B, et al. Introducing intermediate filaments: from discovery to disease[J]. Journal of Clinical Investigation, 2009, 119 (7):1763-1771.

[30] Andrews M, Visser C, Marle-Köster E V. Identification of novel variants for KAP 1.1, KAP 8.1 and KAP 13.3 in South African goats[J]. Small Ruminant Research, 2017, 149:176-180.

[31] Li M N, Liu X, Wang J Q, et al. Molecular characterization of caprine KRTAP13-3 in Liaoning Cashmere goat in China[J]. Journal of Applied Animal Research, 2014, 42(2):140-144.

[32] Biolo $G$, Ciocchi $B$, Lebenstedt $M$, et al. Short-term bed rest impairs amino acid-induced protein anabolism in humans[J]. Journal of Physiology (Oxford), 2004, 558(2):381-388.

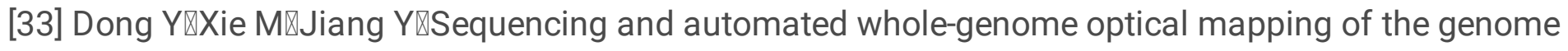
of adomestic goat (Capra hircus)[J]. Nat Biotechnol $₫ 2013 \rrbracket 31(2) \llbracket 135-41 \rrbracket$

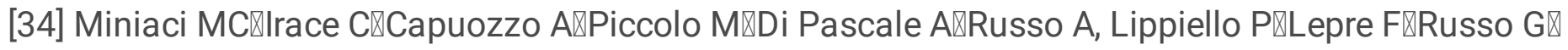
Santamaria R凶Cysteine Prevents the Reduction in Keratin Synthesis Induced by Iron Deficiency in Human Keratinocytes[J].Cell Biochem $₫ 2016 \varangle 117(2) \llbracket 402-12 \rrbracket$ 


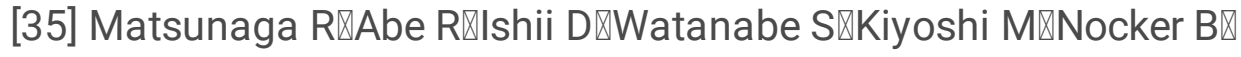

et alषBidirectional binding property of high glycine-tyrosine keratin-associated protein contributes to the mechanical strength and

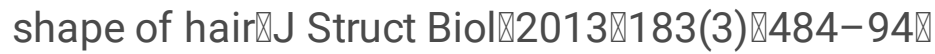

[36] Kehler JS $\triangle D$ David VA囚Schäffer AA et al『Four independent mutations in the feline fibroblast growth factor 5 gene determine the long-haired phenotype in domestic cats $\varangle$ Hered $\varangle 2007 \otimes 98(6) \otimes 555-66 \rrbracket$

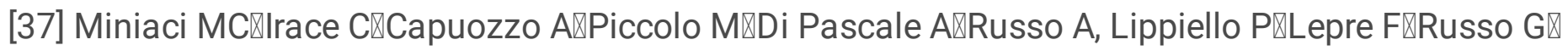
Santamaria R凶Cysteine Prevents the Reduction in Keratin Synthesis Induced by Iron Deficiency in Human Keratinocytes $₫ J$ Cell Biochem $₫ 2016 \otimes 117(2) \llbracket 402-12 \rrbracket$

\section{Figures}

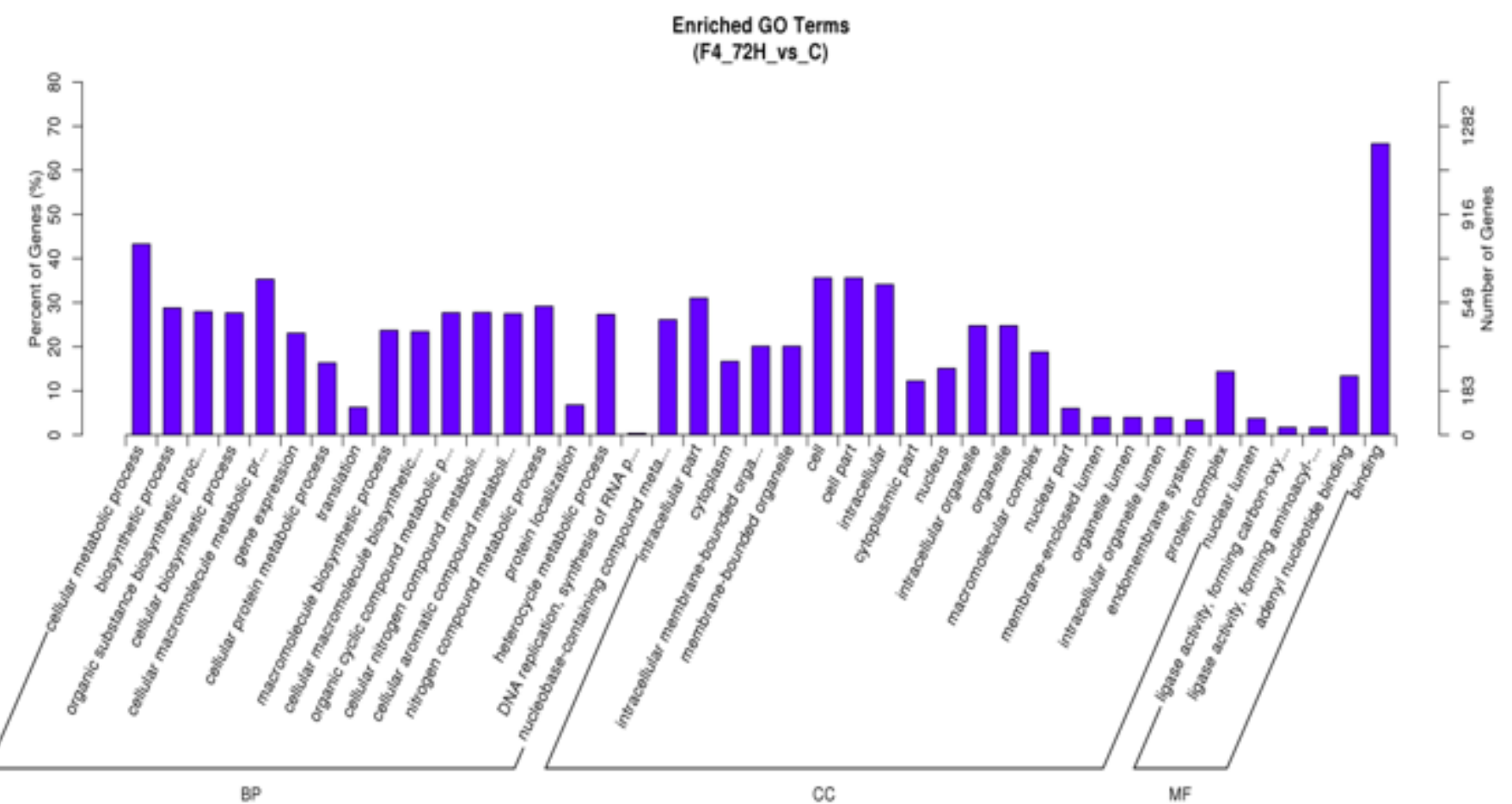

\section{Figure 1}

GO term classification of differentially expressed LncRNA target genes between the F4_72h and C group. Abscissa represents enrichment of the $\mathrm{GO}$ term, ordinate represents the number of target genes in this term and the percentage of target and annotated genes. 


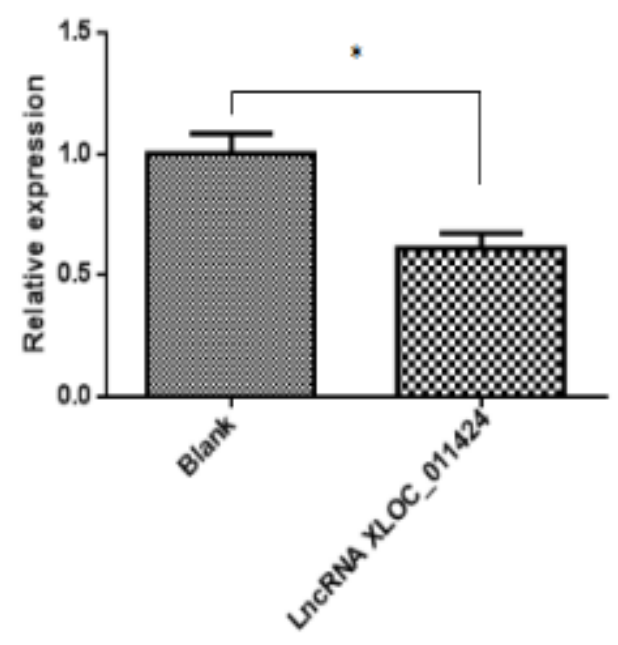

Figure 2

Expression of LncRNA XLOC_011424

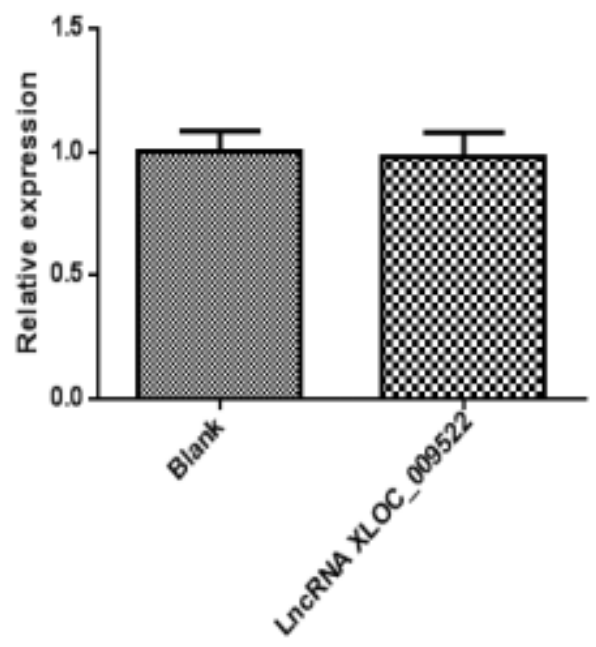

Figure 3

Expression of LncRNA XLOC_009522

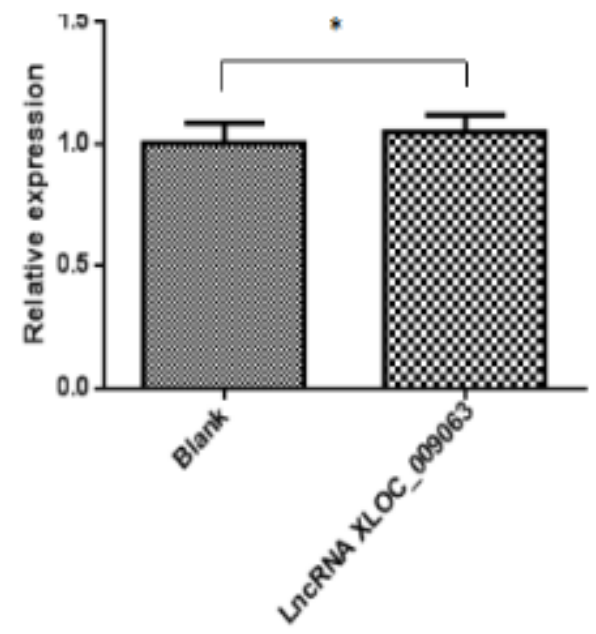


Figure 4

Expression of LncRNA XLOC_009063

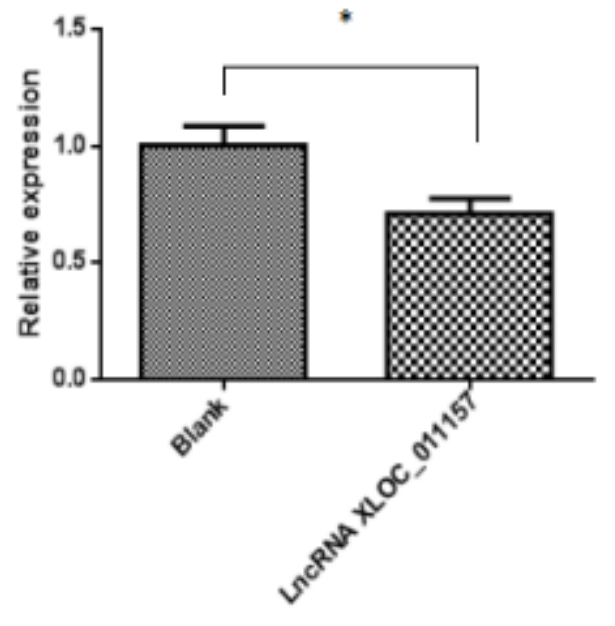

Figure 5

Expression of LncRNA XLOC_011157 * $p<0.05$

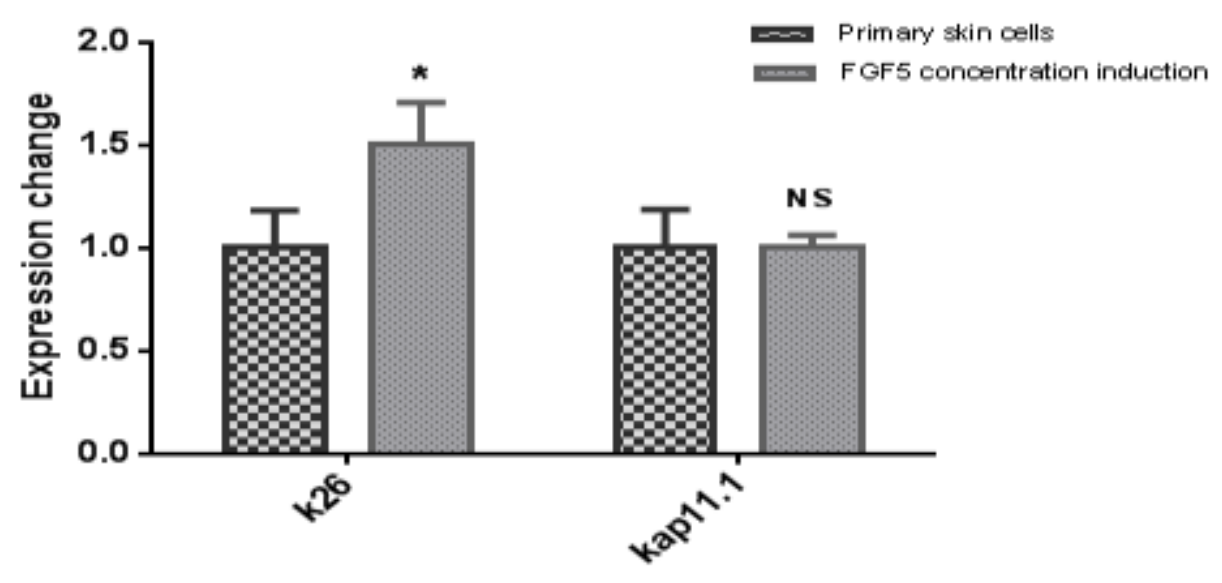

Figure 6

Results of fluorescence quantitative PCR expression of LncRNA keratin gene.Data are the mean \pm SD of three independent experiments, NS (not significant, $P>0.05$ ) 

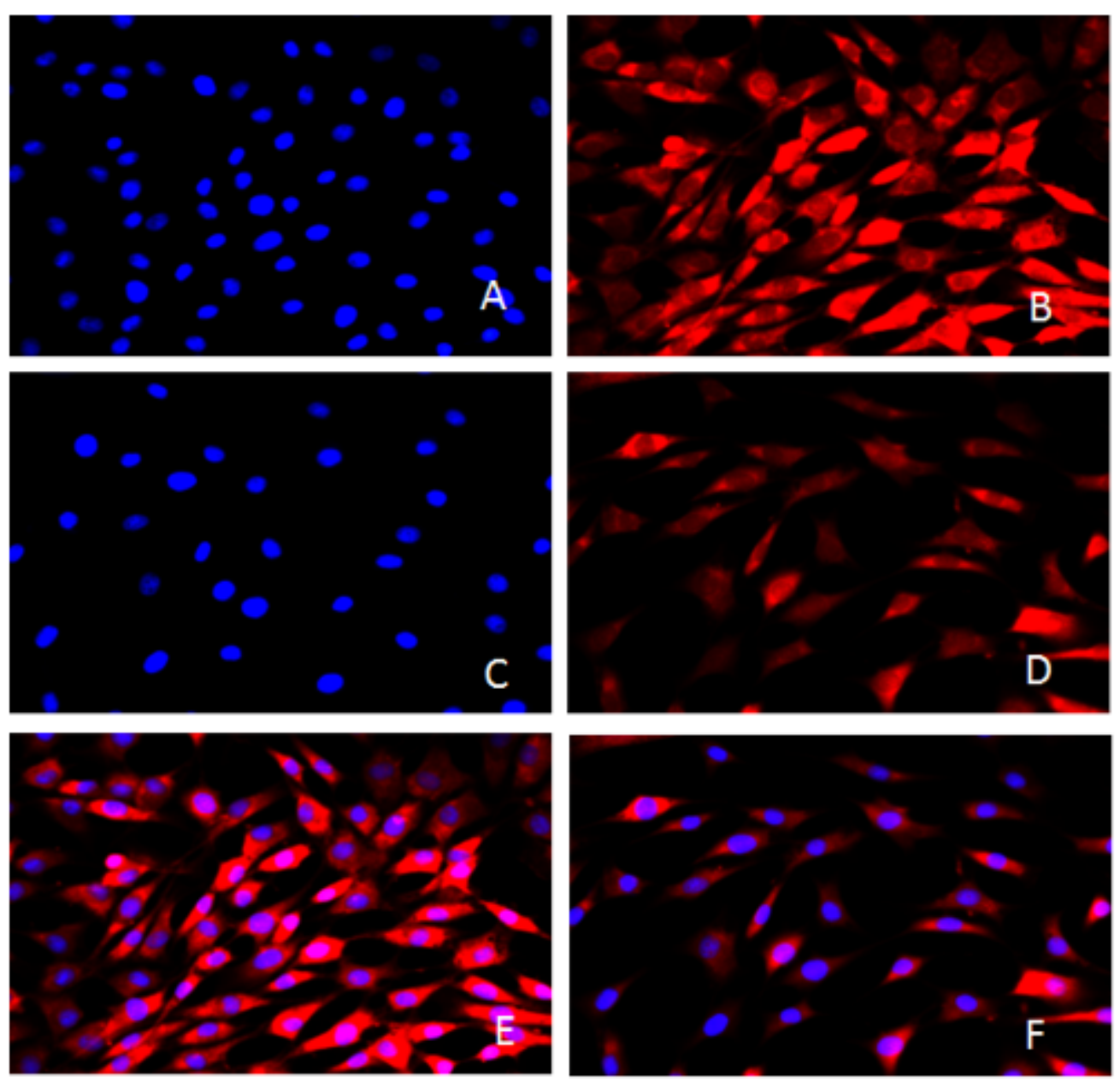

\section{Figure 7}

Intracellular mapping of LncRNA before and after treatment with FGF5. Fig. A shows the location of nuclei in untreated sheep skin cells (40x). Fig. B shows the location of the target LncRNA in untreated sheep skin cells (40x). Fig. Cshows the location of nuclei in sheep skin cells treated with FGF5 (40x). Fig. Dshows the intracellular location of target LncRNA in sheep skin cells treated with FGF5 (40x). Fig. Eis a merge diagram of the nucleus and LncRNA sites in untreated sheep skin cells (40x). Fig. F is a merge diagram (40x) of the nucleus and LncRNA sites in sheep skin cells treated with FGF5.The blue fluorescent stain is nuclear staining, and the red fluorescence represents LncRNA staining. 


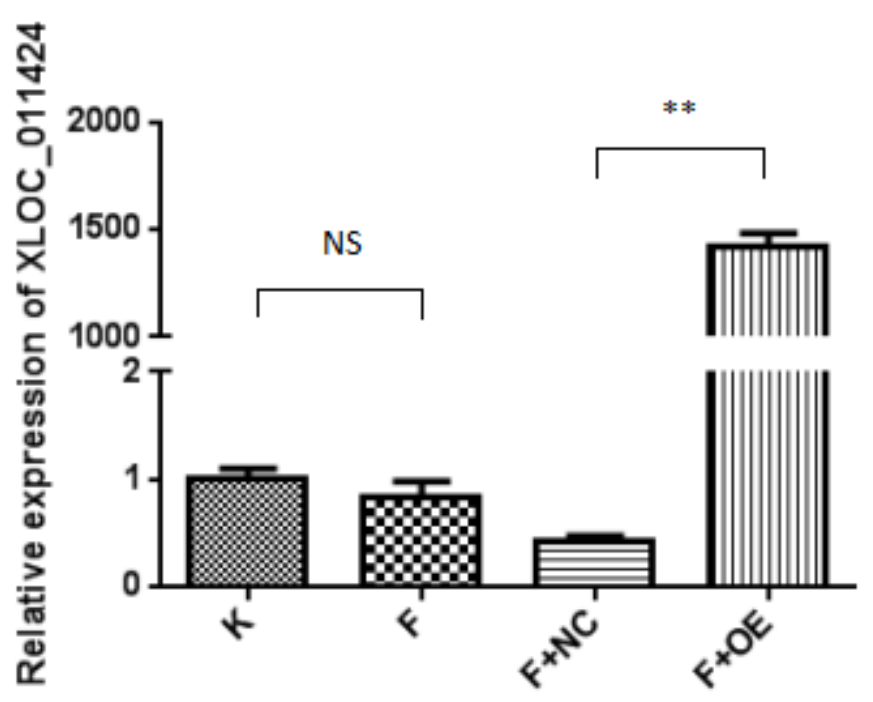

Figure 8

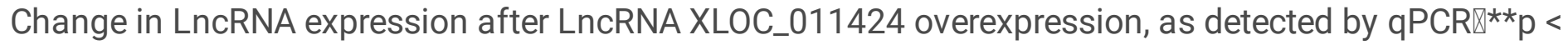
0.01ه.K indicates blank cells, F indicates cells treated with FGF5, F + NC indicates negative control cells treated with FGF5, and F + OE indicates cells overexpressed with LncRNA after FGF5 treatment.Data are the mean \pm SD of three independent experiments, NS (not significant, $P>0.05$ )

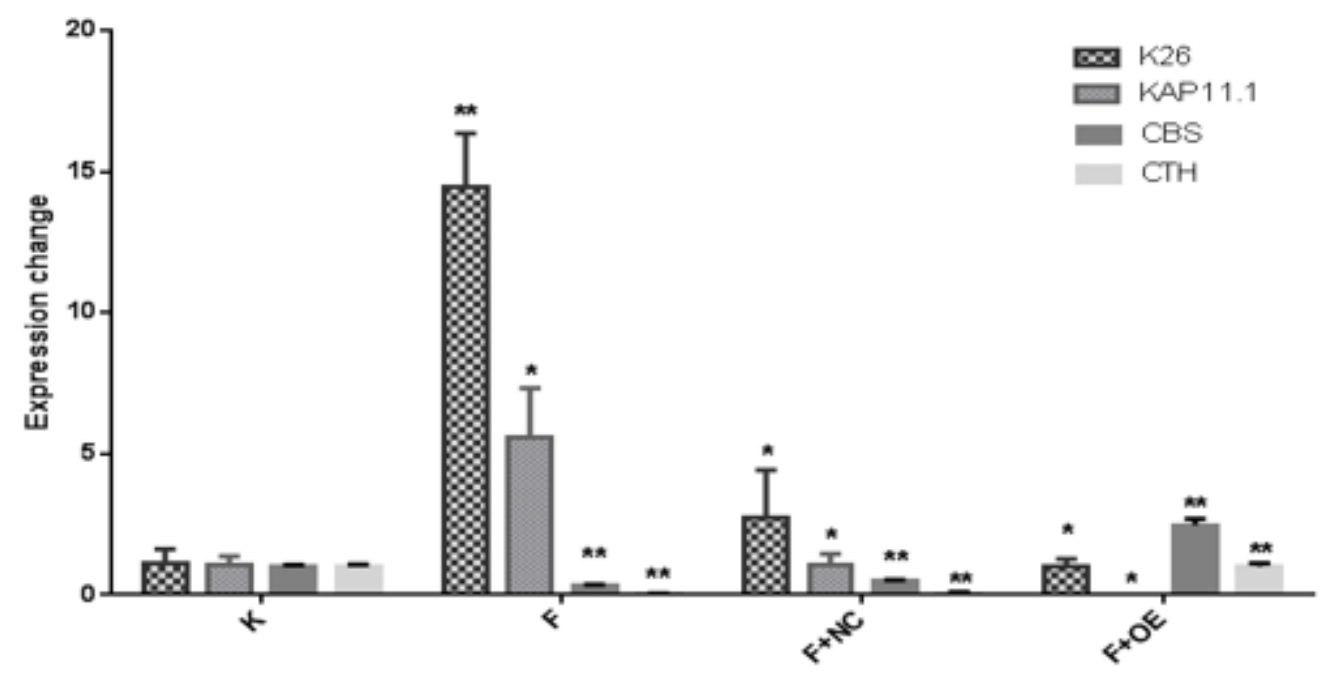

Figure 9

Change in LncRNA keratin gene and target gene expression after LncRNA overexpression, as detected by $q P C R{ }^{\star \star} \mathrm{p}<0.01 \rrbracket$. K indicates blank cells, $\mathrm{F}$ indicates cells treated with FGF5, F + NC indicates negative control cells treated with FGF5, and F + OE indicates cells overexpressed with LncRNA after FGF5 treatment. 


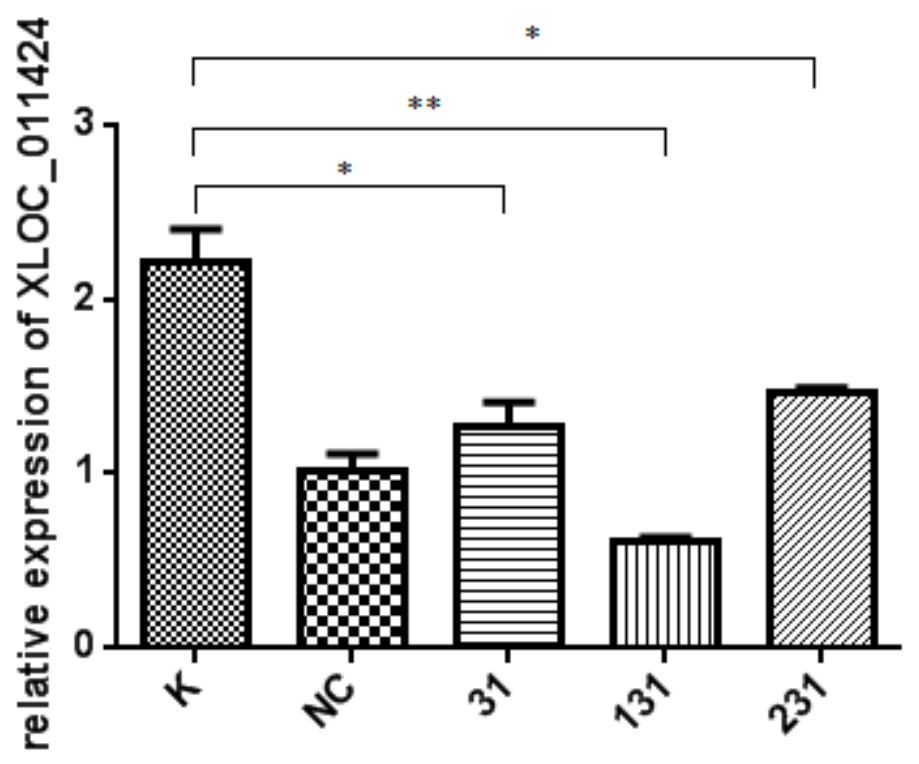

Figure 10

LncRNA gene expression by qPCR. The abscissa is the blank control group, NC is the negative control, and experimental is the group of target spots $31,131,231 * p<0.05 \nabla^{* *} p<0.01$
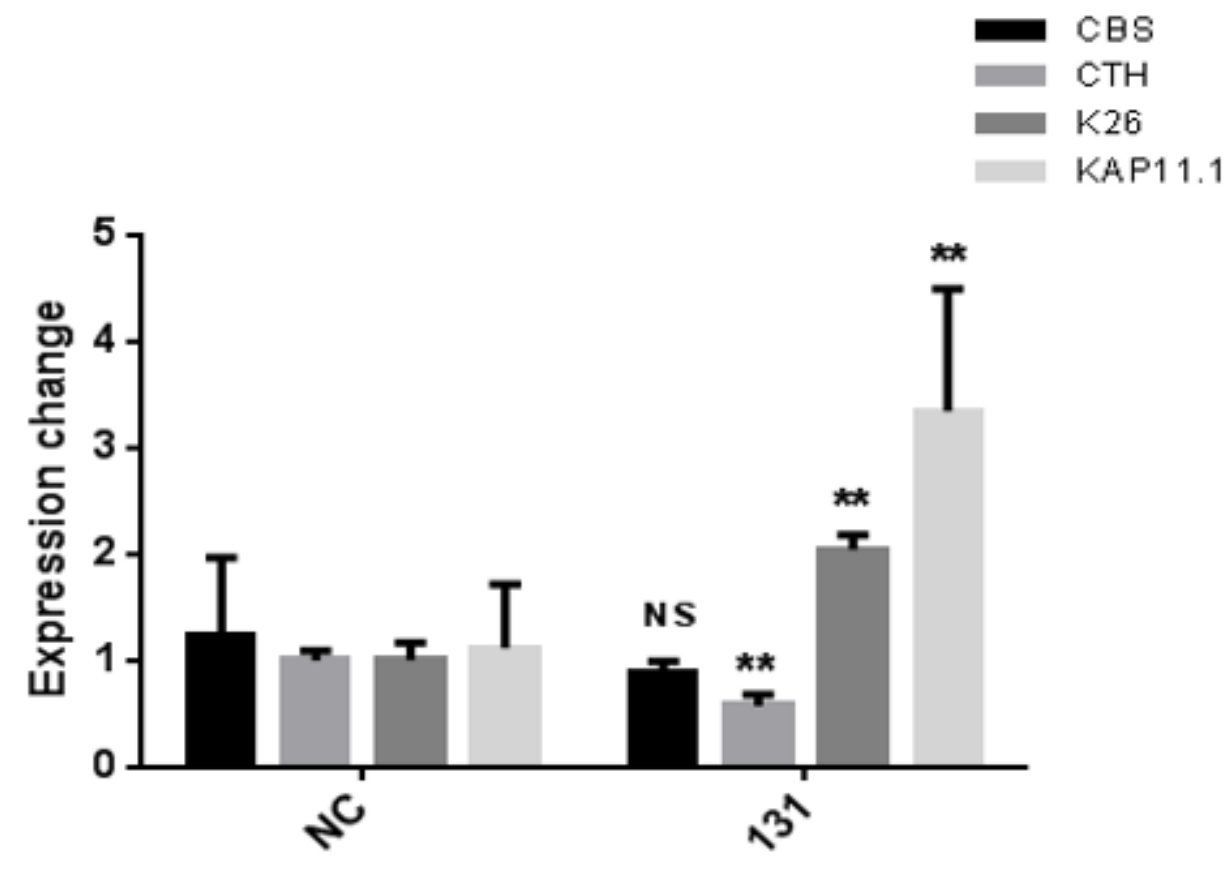

Figure 11

Change in LncRNA keratin gene and target gene expression after LncRNA under-expressionwas detected

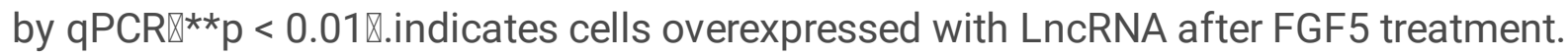

